\title{
Water Resistant Caesium Lead Bromide Crystal Composites
}

\author{
Pedro Urbano Alves, Ian M. Watson, Nicolas Laurand and Martin D. Dawson \\ Institute of Photonics, Department of Physics, SUPA, University of Strathclyde \\ Glasgow, UK \\ pedro.alves@strath.ac.uk
}

\begin{abstract}
Water-resistant luminescent polymer composites with $\mathrm{Cs}_{\mathrm{PbBr}}$ quantum dots in $\mathrm{Cs}_{4} \mathrm{PbBr}_{6}$ crystals $\left(\mathrm{Cs}_{\mathrm{PbBr}} @ \mathrm{Cs}_{4} \mathrm{PbBr}_{6}\right.$ ) are reported. These color-converting materials enable a narrow-band green emission with fast dynamics and are therefore a potential alternative to phosphors, mainly for applications that require a fast modulation of light.
\end{abstract}

Keywords - Visible Light Communication, Perovskite, Quantum Dot, Semiconductor Nanocrystal, Encapsulation, LED.

\section{INTRODUCTION}

Solid-state lighting and display technologies rely on UV/blue GaN LEDs, or in some special cases laser diodes, to optically pump phosphors [1], [2]. For the purpose of high light quality and/or wide color gamut, a combination of narrow-band red and green phosphors are used [3]. For device processing and operation stability, the phosphors are also required to be heat and water resistant [3], [4]. However, the very long photoluminescence (PL) lifetime of phosphors precludes their utilization in applications requiring high speed modulation of the light, e.g. visible light communications [5], [6].

Colloidal quantum dots (QDs) represent an alternative to phosphors for such applications [6], [7]. QDs are nanoscale semiconductor crystals [8] with high PL quantum efficiency, narrow emission linewidth and typical luminescence lifetimes $10^{3}$ to $10^{5}$ shorter than those of phosphors [7], [9]. Perovskite QDs of composition $\mathrm{CsPbX}_{3}$ (with $\mathrm{X}$ a halide ion), demonstrated for the first time 3 years ago [10], are particularly attractive because they have the fastest dynamics of all known QDs [9], [11].

Despite these advantages, QDs have limitations in terms of stability. Several approaches have been reported to improve their thermal stability [12], [13], but their sensitivity to oxygen and moisture continues to be a problem and few solutions have been reported to solve them [13]-[15].

A promising material for narrow-band green color conversion is the so-called $\mathrm{CsPbBr}_{3} @ \mathrm{Cs}_{4} \mathrm{PbBr}_{6}$ [16]. It consists of $\mathrm{CsPbBr}_{3}$ QDs formed within the wide band gap material $\mathrm{Cs}_{4} \mathrm{PbBr}_{6}$, which acts as a matrix for the QDs [17], [18]. As opposed to colloidal QDs, these $\mathrm{CsPbBr}_{3}$ QDs are inherently encapsulated in $\mathrm{Cs}_{4} \mathrm{PbBr}_{6}$ and have no organic ligands on their surfaces, which is beneficial for thermal and photostability. The band edge emission of such material has been shown to be practically temperature invariant [16]. However, a low cost method to enhance the stability of $\mathrm{CsPbBr}_{3} @ \mathrm{Cs}_{4} \mathrm{PbBr}_{6}$ upon water exposure remains a challenge. This work comprises synthesis and analysis of $\mathrm{CsPbBr}_{3} @ \mathrm{Cs}_{4} \mathrm{PbBr}_{6}$ crystals, and studies of the luminescence properties of related composite samples when placed in contact with water. A guest-host approach is chosen and two different polymeric materials are studied for the matrix host: poly(methyl methacrylate) (PMMA) and polydimethylsiloxane (PDMS).

\section{MAterials AND Methodology}

\section{A. Synthesis of Caesium Lead Bromide Crystals}

Stoichiometric amounts of caesium bromide and lead bromide at a 4:1 mole ratio were dissolved together in a minimum quantity of dimethylsulfoxide solvent for the $\mathrm{CsPbBr}_{3} @ \mathrm{Cs}_{4} \mathrm{PbBr}_{6}$ preparation. This precursor solution was placed in an open inner vial inside a sealed outer jar containing chloroform. Vapour diffusion of the chloroform into the metal salt solution was complete over about 24 hours and caused formation and precipitation of the crystalline solids. This method follows previously reported preparations, but with chloroform acting as an antisolvent [16]. The collected crystals, with sizes ranging from a few hundreds of $\mu \mathrm{m}$ to $\mathrm{mm}$, were washed with chloroform and dried under atmospheric pressure at $60{ }^{\circ} \mathrm{C}$ (Fig. 1). Manual grinding was done with an agate pestle and mortar.

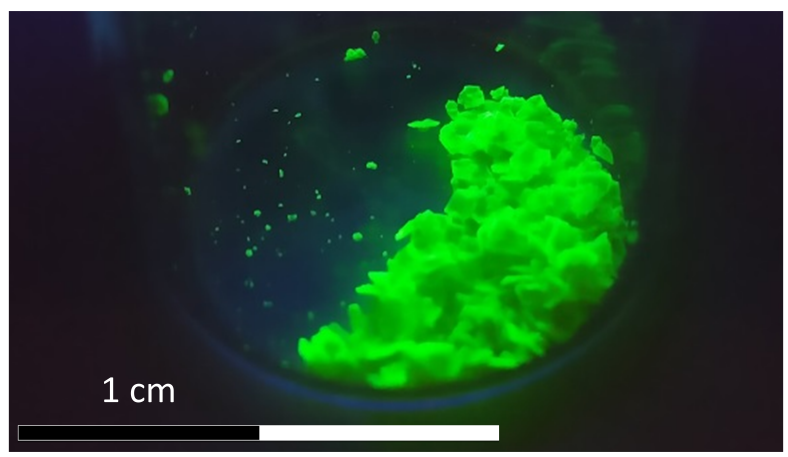

Fig. 1. Synthesized bulk $\mathrm{CsPbr}_{3} @ \mathrm{Cs}_{4} \mathrm{PbBr}_{6}$ crystals under UV illumination.

\section{B. Preparation of Composites}

Two PMMA samples were fabricated by dissolving and stirring PMMA (Sigma-Aldrich) in toluene, together with ground $\mathrm{CsPbBr}_{3} @ \mathrm{Cs}_{4} \mathrm{PbBr}_{6}$. A mass ratio of 3:1 was used for PMMA and crystals. The solvent was left to evaporate over time, under atmospheric pressure and temperature. One of the PMMA samples, a slab with a thickness of approximately $1 \mathrm{~mm}$, was tested as it was after the PMMA polymerization (PMMA slab). The second sample, also a slab with a thickness of approximately $1 \mathrm{~mm}$, was additionally 
encapsulated in PDMS (PMMA slab encapsulated in PDMS).

Two further PDMS samples were prepared by stirring PDMS (Sylgard 184, Dow Corning) with ground $\mathrm{CsPbBr}_{3} @ \mathrm{Cs}_{4} \mathrm{PbBr}_{6}$. A mass ratio of 3:1 was used between PDMS and crystals, and a mass ratio of $5: 1$ between elastomer and curing agent. These composites were cured at $60^{\circ} \mathrm{C}$. One of the samples was moulded in the shape of a slab, approximately $1 \mathrm{~mm}$ thick (PDMS slab), and the other in the shape of a thicker composite, approximately $3 \mathrm{~mm}$ thick (PDMS thick). Crystal sedimentation was observed during the preparation of PDMS samples. This led to a heterogeneity in the concentration of $\mathrm{CsPbBr}_{3} @ \mathrm{Cs}_{4} \mathrm{PbBr}_{6}$ in the PDMS slab and to a lower concentration of $\mathrm{CsPbBr}_{3} @ \mathrm{Cs}_{4} \mathrm{PbBr}_{6}$ in the PDMS thick sample.

\section{Phase Analysis of Perovskite Crystals}

$\mathrm{X}$-Ray Difraction (XRD) analysis was performed on ground $\mathrm{CsPbBr}_{3} @ \mathrm{Cs}_{4} \mathrm{PbBr}_{6}$ crystals using a Bruker D8 Discover diffractometer (GX000915), and copper radiation at a wavelength of $1.5406 \AA$.

\section{Characterization of Composites}

A $100 \times 100 \mu \mathrm{m}^{2} 450 \mathrm{~nm}$-emitting LED was used as pump source to measure the PL of all composites. Two sets of two lenses each (aspheric lenses, Numerical Aperture, NA=0.621 and diameter of $45 \mathrm{~mm}$, Thorlabs) were used to, respectively, image the LED light onto the samples, and to collect the composite's emission onto the detector. A fiber-coupled CCD spectrometer (Ocean Optics, USB 4000; resolution: $2 \mathrm{~nm}$ at full width half maximum) was used to acquire the emitted spectral data.

The absorbance was measured by replacing the pump source in the previously described setup by a tungsten halogen lamp (Ocean Optics, HL-2000). Blank composites (i.e. with no $\mathrm{CsPbBr}_{3} @ \mathrm{Cs}_{4} \mathrm{PbBr}_{6}$ crystals) of PDMS and PMMA were used as reference samples.

\section{RESULTS AND DISCUSSION}

\section{A. XRD on $\mathrm{CsPbBr}_{3} @ \mathrm{Cs}_{4} \mathrm{PbBr}_{6}$ crystals}

Fig. 2 shows a powder diffraction pattern from a ground portion of the $\mathrm{CsPbBr}_{3} @ \mathrm{Cs}_{4} \mathrm{PbBr}_{6}$ crystal preparation with nominal $\mathrm{Cs}_{4} \mathrm{PbBr}_{6}$ stoichiometry, compared with a stick-plot representation of the literature pattern of rhombohedral $\mathrm{Cs}_{4} \mathrm{PbBr}_{6}$ [16]. A log scale has been used for clear presentation of weaker peaks. Positions of stronger peaks from our preparation match those expected from the $\mathrm{Cs}_{4} \mathrm{PbBr}_{6}$ phase, although there is evidence of preferred orientation effects on relative peak intensities, and one peak attributable to $\mathrm{CsBr}$ starting material. Fig. 2 is also labelled with peak positions calculated for cubic, perovskite-phase $\mathrm{CsPbBr}_{3}$ with a lattice constant of $5.84 \AA$, and there are clear matches for the 100, 110 and 210 reflections. The $\mathrm{CsPbBr}_{3}$ 111 reflection cannot be observed, but is weak in reported powder patterns of bulk material [19]. The findings just discussed are consistent with those of previous authors who have presented XRD evidence for spontaneous formation of $\mathrm{CsPbBr}_{3}$ nanocrystals within a bulk crystalline $\mathrm{Cs}_{4} \mathrm{PbBr}_{6}$ host, and attributed green luminescence to this nanophase [16]-[18].

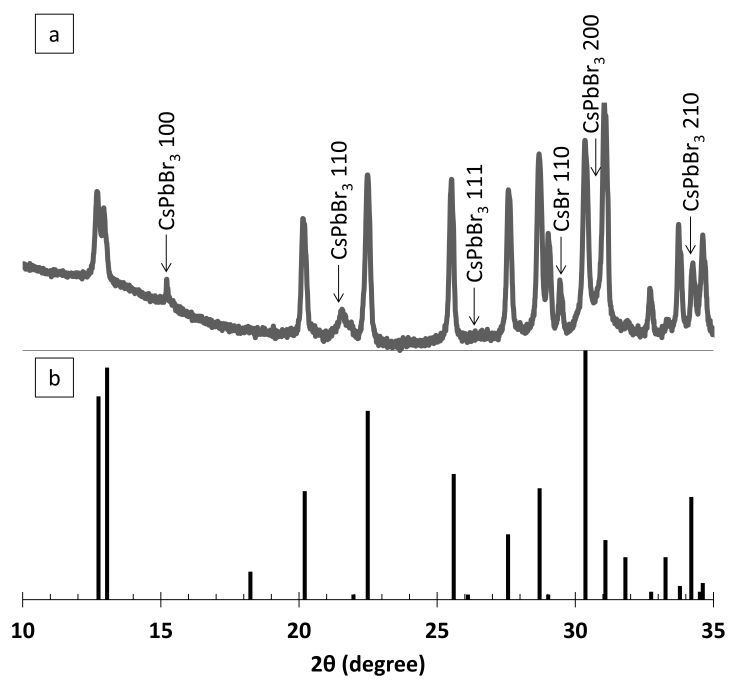

Fig. 2. a) Log-scaled powder XRD pattern from the $\mathrm{CsPbBr}_{3} @ \mathrm{Cs}_{4} \mathrm{PbBr}_{6}$ crystal preparation with nominal $\mathrm{Cs}_{4} \mathrm{PbBr}_{6}$ stoichiometry. The intensity range in the original dataset was 1-100 counts per second. b) Stick plot representation of literature data for the bulk $\mathrm{Cs}_{4} \mathrm{PbBr}_{6}$ phase, from [16].

\section{B. Polymer/CsPbBr ${ }_{3} @ \mathrm{Cs}_{4} \mathrm{PbBr}_{6}$ Composites}

Four different samples, using PMMA or PDMS host matrices, had their PL and absorbance measured before full immersion in deionised water. The PL spectrum of each sample prior to immersion in water can be seen in Fig. 3. There are differences in the initial maximum intensity peaks and respective emission wavelengths between samples. This is explained by intrinsic variations in the concentration of $\mathrm{CsPbr}_{3} @ \mathrm{Cs}_{4} \mathrm{PbBr}_{6}$ crystals and the different thickness of samples. Because the intrinsic PL overlaps with the absorption spectrum, such differences lead to different amounts of reabsorption, hence different intensities and red shifts in the PL spectrum. The PDMS thick, which has the least re-absorption, has a PL peak very close to the intrinsic $\mathrm{CsPbBr}_{3}$ emission at $520 \mathrm{~nm}$. The typical PL linewidth is 17 $\pm 2 \mathrm{~nm}$ at full width half maximum. PL and absorbance acquisitions were repeated 1,4 and 72 hours after the samples had been thoroughly stirred underwater.

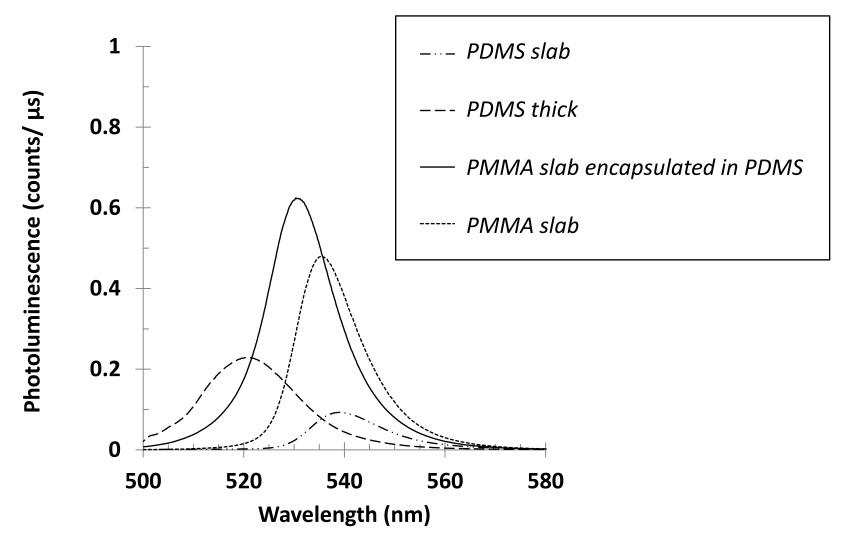

Fig. 3. Photoluminescence measurements of each of the fabricated samples prior to their submersion in water. Pumping was by a $450 \mathrm{~nm} \mu \mathrm{LED}$.

The PL intensity of PMMA composites was constant over time, and its subsequent encapsulation with PDMS showed little to no extra improvement (Fig. 4).

On the other hand, the PDMS samples showed an adverse effect as seen from the PL characteristics after 
exposure to water. After 4 hours underwater, the PDMS slab (PDMS thick) had its PL intensity reduced by almost two orders (one order) of magnitude. These results suggest that water was able to reach and affect most of the crystals in the PDMS samples, despite the hydrophobicity of PDMS.

The shifts of the PL peak wavelength and of the absorption spectrum after 1, 4 and 72-hour-immersion (with respect to the data before immersion) are plotted for all samples in Fig. 5. The PL and absorption of PDMS samples red shifted by approximately $8 \mathrm{~nm}$. This is significant and indicates a reduction of the confinement of the $\mathrm{CsPbBr}_{3}$ QDs. Although superficially suggestive of an increased particle size of the $\mathrm{CsPbBr}_{3}$ QDs, other possibilities are compositional changes to their surface layers and/or adjacent $\mathrm{Cs}_{4} \mathrm{PbBr}_{6}$ host material. There is a smaller shift for the PMMA samples (1 to $3 \mathrm{~nm}$ ), very close to the measurement error.

As the wavelength increases, the absorbance decreases for all samples. Its magnitude, however, suggests that samples show scattering, and they become even more strongly scattering after being put into water.

This increase in absorbance after being in contact with water, and a red shift of the emission spectrum, suggest that samples underwent substantial physical and chemical changes while exposed to water. While these effects are negligible in PMMA, PDMS samples presented a significant drop in their PL. A possible explanation might arise from the differences in polymer chain arrangements and consequent water sorption properties between PDMS and PMMA. While PMMA has predominantly linear chains, and forms a dense, glassy microstructure after solvent evaporation, the PDMS used is engineered to partially cross-link during curing, to give an elastomeric end product [20], [21]. This characteristic of PDMS appears to give a microstructure allowing more permeation of water into the composites, and degradation of the luminescent $\mathrm{CsPbBr}_{3}$ QDs. Water permeation in the two polymer hosts have been extensively studied in other contexts, and there is quantitative support for these findings on the relative properties of PDMS [20] and PMMA [22].

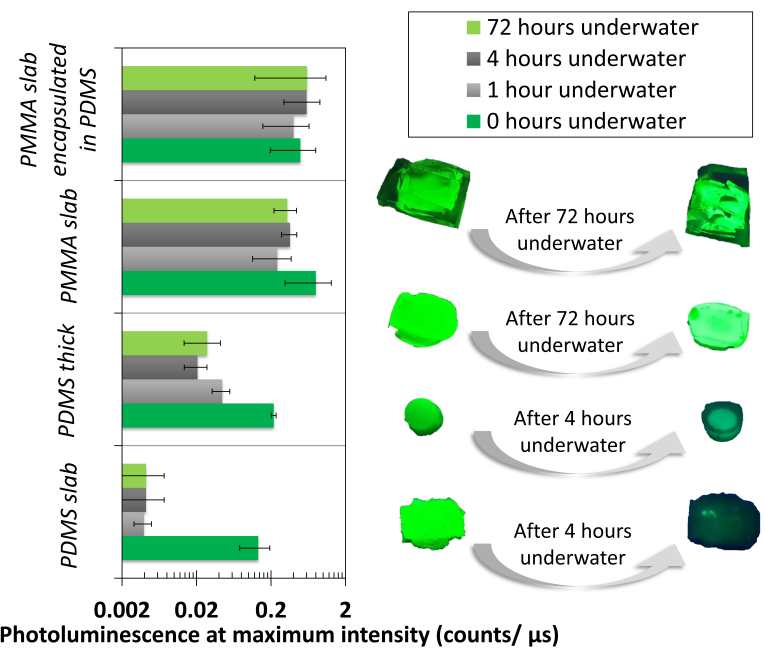

Fig. 4. Photoluminescence at maximum intensity over time, for each of samples studied. Samples are presented in the same order as in the bar plot.

The PL variations over time (Fig. 4) are in agreement with the thickness of the polymer host matrix, and the relative permeability to water just proposed. The PDMS slab thickness was small enough to have most of its content of luminescent crystals affected, and its intensity reduced by two orders of magnitude. The PDMS thick had its PL peak decreased by one order of magnitude, suggesting that the innermost crystals were more protected against water. PMMA samples showed little to no fluctuation on their maximum intensity PL peaks. Such results suggest that only the crystals exposed on the surface the samples might have been affected, and that crystals are better protected against water when embedded in PMMA.

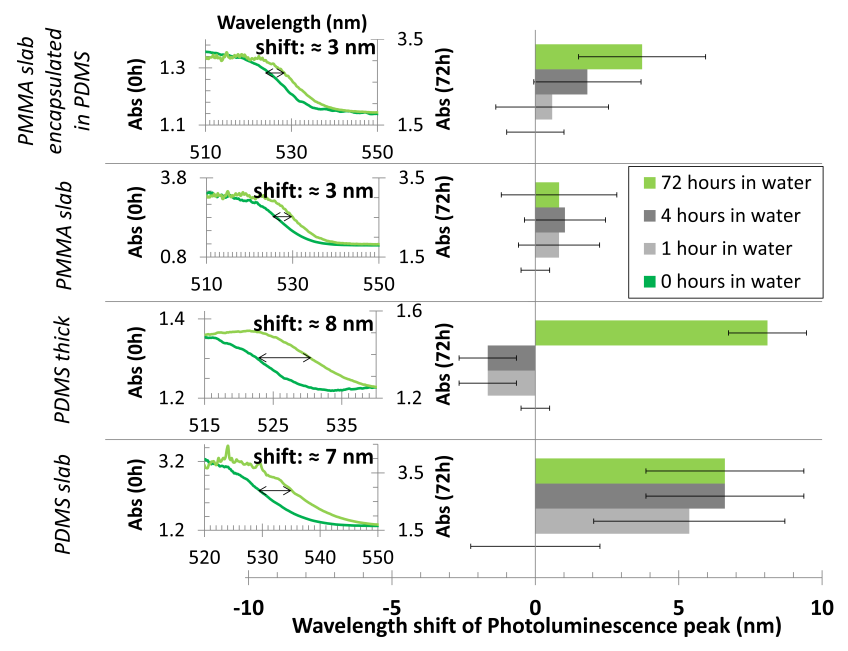

Fig. 5. Shift in wavelength of the photoluminescence peak of each sample after $0,1,4$ and 72 hour-immersion in water (right) with (left) absorbance spectra before and after immersion in water for 72 hours.

\section{CONCLUSIONS}

Motivated by their low manufacturing cost and simple processing, PDMS and PMMA were tested and studied as host matrices for the $\mathrm{CsPbBr}_{3} @ \mathrm{Cs}_{4} \mathrm{PbBr}_{6}$ crystals synthesized in this work.

The PDMS samples were not stable upon immersion in water. This finding indicates that water was able to reach the guest $\mathrm{CsPbBr}_{3} @ \mathrm{Cs}_{4} \mathrm{PbBr}_{6}$ crystals. On the other hand PMMA composites showed little to no trace of degradation when immersed in water under vigorous stirring for up to 72 hours.

PMMA was found in this work to be an effective matrix host for $\mathrm{CsPbBr}_{3} @ \mathrm{Cs}_{4} \mathrm{PbBr}_{6}$. Its encapsulation properties show potential for macrosized industrial applications, such as color conversion, where the degradation at the surface is mitigated by the extremely low water penetration depth, and the volume of the composite itself. This polymer matrix approach to minimise moisture-sensitivity should therefore enable the utilisation of the attractive fast color converting properties of $\mathrm{CsPbr}_{3} @ \mathrm{Cs}_{4} \mathrm{PbBr}_{6}$ for practical applications, such as underwater communication.

\section{ACKNOWLEDGMENT}

We acknowledge support from the EPSRC Platform Grant EP/P0274X/2. We thank Alan Martin for X-ray powder diffraction, which was performed in the CMAC National Facility, University of Strathclyde Technology and 
Innovation Centre, funded with a UKRPIF capital award, SFC ref. H13054, from HEFCE.

Supporting data (dataset) can be found at: http://dx.doi.org/10.15129/fb9f6aa0-26e2-419a-8788c2b051453108

\section{REFERENCES}

[1] M. R. Krames et al., "Status and future of high-power lightemitting diodes for solid-state lighting," IEEE/OSA J. Disp. Technol., vol. 3, no. 2, pp. 160-175, 2007.

[2] J. J. Wierer, J. Y. Tsao, and D. S. Sizov, "Comparison between blue lasers and light-emitting diodes for future solid-state lighting," Laser Photonics Rev., vol. 7, no. 6, pp. 963-993, 2013.

[3] L. Wang et al., "Highly efficient narrow-band green and red phosphors enabling wider color-gamut LED backlight for more brilliant displays," Opt. Express, vol. 23, no. 22, p. 28707, 2015.

[4] L. Huang et al., "Highly Stable $\mathrm{K}_{2} \mathrm{SiF}_{6}: \mathrm{Mn}^{4+} @ \mathrm{~K}_{2} \mathrm{SiF}_{6}$ Composite Phosphor with Narrow Red Emission for White LEDs," ACS Appl. Mater. Interfaces, vol. 10, no. 21, pp. 18082-18092, 2018.

[5] D. C. O'Brien, L. Zeng, H. Le-Minh, G. Faulkner, J. W. Walewski, and S. Randel, "Visible Light Communications: Challenges and possibilities," IEEE Int. Symp. Pers. Indoor Mob. Radio Commun. PIMRC, pp. 1-5, 2008.

[6] N. Laurand et al., "Colloidal quantum dot nanocomposites for visible wavelength conversion of modulated optical signals," Opt. Mater. Express, vol. 2, no. 3, pp. 250-260, 2012.

[7] M. F. Leitao et al., "Gb/s Visible Light Communications with Colloidal Quantum Dot Color Converters," IEEE J. Sel. Top. Quantum Electron., vol. 23, no. 5, 2017.

[8] J. Y. Kim, O. Voznyy, D. Zhitomirsky, and E. H. Sargent, "25th anniversary article: Colloidal quantum dot materials and devices: A quarter-century of advances," Adv. Mater., vol. 25, no. 36, pp. 4986-5010, 2013.

[9] F. T. Rabouw and C. de Mello Donega, "Excited-State Dynamics in Colloidal Semiconductor Nanocrystals," Top. Curr. Chem., vol. 374 , no. 5, pp. 1-30, 2016.

[10] L. Protesescu et al., "Nanocrystals of Cesium Lead Halide Perovskites $\left(\mathrm{CsPbX}_{3}, \mathrm{X}=\mathrm{Cl}, \mathrm{Br}\right.$, and I): Novel Optoelectronic Materials Showing Bright Emission with Wide Color Gamut," Nano Lett., vol. 15, no. 6, pp. 3692-3696, 2015.

[11] M. A. Becker et al., "Bright triplet excitons in caesium lead halide perovskites," Nature, vol. 553, no. 7687, pp. 189-193, 2018.

[12] B. T. Diroll, G. Nedelcu, M. V. Kovalenko, and R. D. Schaller, "High-Temperature Photoluminescence of $\mathrm{CsPbX}_{3}(\mathrm{X}=\mathrm{Cl}, \mathrm{Br}, \mathrm{I})$ Nanocrystals," Adv. Funct. Mater., vol. 27, no. 21, pp. 1-7, 2017.

[13] A. Loiudice, S. Saris, E. Oveisi, D. T. L. Alexander, and R. Buonsanti, " $\mathrm{CsPbBr}{ }_{3} \mathrm{QD} / \mathrm{AlO}_{\mathrm{x}}$ Inorganic Nanocomposites with Exceptional Stability in Water, Light, and Heat," Angew. Chemie - Int. Ed., vol. 56, no. 36, pp. 10696-10701, 2017.

[14] J. Ren, X. Dong, G. Zhang, T. Li, and Y. Wang, "Air-stable and water-resistant all-inorganic perovskite quantum dot films for white-light-emitting applications," New J. Chem., vol. 41, no. 22, pp. 13961-13967, 2017.

[15] L. Gomez, C. De Weerd, J. L. Hueso, and T. Gregorkiewicz, "Color-stable water-dispersed cesium lead halide perovskite nanocrystals," Nanoscale, vol. 9, no. 2, pp. 631-636, 2017.

[16] Y. Wang et al., "Solution-Grown $\mathrm{CsPbBr}_{3} / \mathrm{Cs}_{4} \mathrm{PbBr}_{6}$ Perovskite Nanocomposites: Toward Temperature-Insensitive Optical Gain," Small, vol. 13, no. 34, pp. 1-8, 2017.

[17] X. Chen et al., "Centimeter-Sized $\mathrm{Cs}_{4} \mathrm{PbBr}_{6}$ Crystals with Embedded $\mathrm{CsPbBr}_{3}$ Nanocrystals Showing Superior Photoluminescence: Nonstoichiometry Induced Transformation and Light-Emitting Applications," Adv. Funct. Mater., vol. 28, no. 16 , pp. 1-7, 2018.

[18] L. N. Quan et al., "Highly Emissive Green Perovskite Nanocrystals in a Solid State Crystalline Matrix," Adv. Mater., vol. 29 , no. 21 , pp. 1-6, 2017.

[19] C. C. Stoumpos et al., "Crystal growth of the perovskite semiconductor $\mathrm{CsPbBr}_{3}$ : A new material for high-energy radiation detection," Cryst. Growth Des., vol. 13, no. 7, pp. 2722-2727, 2013.

[20] S. J. Harley, E. A. Glascoe, and R. S. Maxwell, "Thermodynamic study on dynamic water vapor sorption in Sylgard-184," J. Phys. Chem. B, vol. 116, no. 48, pp. 14183-14190, 2012.

[21] P. J. Flory, Principles of Polymer Chemistry. Ithaca, New York: Cornell University Press, pp. 45-52, 1953.

[22] M. Unemori, Y. Matsuya, S. Matsuya, A. Akashi, and A. Akamine, "Water absorption of poly(methyl methacrylate) containing 4-methacryloxyethyl trimellitic anhydride," Biomaterials, vol. 24, no. 8, pp. 1381-1387, 2003. 